\title{
A Taylor Series based Fuzzy Mathematical Approach for Multi Objective Linear Fractional Programming Problem with Fuzzy Parameters
}

\author{
Surapati Pramanik \\ Department of Mathematics, \\ Nandalal Ghosh B.T. \\ College, Panpur, P.O.- \\ Narayanpur, District-North \\ 24 Parganas, Pin Code- \\ 743126, West Bengal, India
}

\author{
Indrani Maiti \\ Department of Mathematics, \\ National Institute of Technology, \\ Jamshedpur \\ Jharkhand-831014, India
}

\author{
Tarni Mandal \\ Department of Mathematics, \\ National Institute of Technology, \\ Jamshedpur \\ Jharkhand-831014, India
}

\begin{abstract}
This article presents an approach to acquire the solution of multi-objective linear fractional programming problems where the parameters are assumed to be triangular fuzzy numbers. This is done through a fuzzy mathematical programming perspective based on an approximation method using Taylor series. The problem is first formulated into an equivalent deterministic form using the concept of $\alpha$-cuts. The associated membership function of each objective function is formulated using the individual optimal solution and is then converted into a linear function by applying the first order Taylor series. The multi-objective linear fractional programming problem then gets reduced to a linear programming problem by applying fuzzy mathematical programming. To illustrate the computational simplicity and applicability of the proposed approach, a numerical example is solved and the results are compared with existing methods.
\end{abstract}

\section{General Terms}

Multi-objective fuzzy linear fractional programming

\section{Keywords}

Multi-objective linear fractional programming problem, fuzzy mathematical programming, Taylor series, triangular fuzzy number, $\alpha$-cut

\section{INTRODUCTION}

Fractional programming refers to a special class of nonlinear optimization problem where the objective function is in the form of a ratio of two functions. The importance of this type of problems arises as there are many cases in business, finance, engineering etc. where we need to optimize the ratio of two quantities such as raw materials/output, cost/profit, nurse/patient, stock/sales etc. So fractional programming becomes an indispensable tool in such situations and this leads to researches to find suitable methods to solve them. linear fractional programming is a particular case of fractional programming, where the objective function is a ratio of two linear functions. Various methods have been formulated by many researchers like Isbell and Marlow [1], Charnes and Cooper [2], Dinklebach [3], Schaible [4], Gilmore and Gomory [5] and Swarup [6] to solve fractional programming and linear fractional programming.

In practical life, there often arises situation where we need to optimize more than one objective function. This leads to the extension of linear fractional programming to Multi-Objective
Linear Fractional Programming (MOLFP) problem. Several methodologies have been proposed in the literature to solve MOLFP problems. A goal programming approach was proposed by Kornbluth and Steur [7] to solve MOLFP problem. Nykowski and Zolkiewski [8] developed a compromise approach for MOLFP problems. Dey and Pramanik [9] developed a goal programming approach to solve MOLFP problems in crisp environment by employing first order Taylor series approximation.

Introduction of fuzzy set theory [10] to deal optimization problem provides a new insight to solve MOLFP problems. Several methods such as linguistic approach [11] based on the work of Zadeh [12-14], fuzzy goal programming approach [15], an interactive fuzzy satisfying method [16], a fuzzy set theoretic method [17] were proposed to solve MOLFP problems. Chakraborty and Gupta [18] presented MOLFP problems by suitably transforming it to multi-objective linear programming problems through the transformation of variables. Guzel and Shivari [19] discussed the solution of MOLFP problems using Taylor series. Toksari [20] employed a Taylor series based fuzzy goal programming technique to solve MOLFP problems.

Pramanik and Roy [21, 22] presented fuzzy goal programming using negative deviational variables only in their formulation. Pramanik and Roy [23, 24] presented a fuzzy goal programming model with fuzzy parameters. Pramanik $[25,26]$ presented bi-level programming problem and multi-level programming problem with fuzzy parameters respectively using fuzzy goal programming approach. Pramanik et al. [27, 28] studied bi-level multi-objective programming problem and decentralized bi-level multiobjective programming problem with fuzzy parameters using fuzzy goal programming approach.

Pramanik and Dey [29] presented an MOLFP using fuzzy goal programming model. Pramanik and Dey [30] also presented a priority based fuzzy goal programming approach to solve MOLFP problems. Pramanik and Dey [31, 32, 33, 34] employed first order Taylor polynomial series to solve bilevel linear fractional programming problem, bi-level MOLFP problems, linear fractional bi-level decentralized programming problem, multilevel fractional programming problem, respectively. Dey et al. [35] developed the Technique for Order of Preference by Similarity to Ideal Solution (TOPSIS) approach to solve bi-level MOLFP problem. 
Banerjee and Pramanik [36] developed goal programming approach to chance constrained MOLFP problem based on Taylor's series approximation. Pramanik \& Dey [37] presented multi-objective linear plus linear fractional programming problem based on Taylor series approximation. Pramanik et al. [38] developed multi-level multi-objective linear plus linear fractional programming problem based on FGP approach. Banerjee and Pramanik [39] presented chance constrained multi-objective linear plus linear fractional programming problem based on Taylor's series approximation. Pramanik et al. [40] formulated chance constrained linear plus linear fractional bi-level programming problem. Several studies have been made using Taylor's polynomial series approximation to deal quadratic programming problem [41, 42], quadratic bi-level programming problem [43], quadratic bi-level multi-objective programming problem [44] and Chance constrained quadratic bi-level programming problem [45].

In real life situations, the parameters involved in a MOLFP problem such as the coefficients of the objective function and the constraints, may not be precisely known as various factors are associated with them. Error may occur while collecting data or data may not be precisely known. Fluctuating market conditions may produce difficulties in representing the parameters as fixed numbers. To deal with such kind of imprecise situation, MOLFP problems with fuzzy parameters were introduced which are referred to as Multi Objective Fuzzy Linear Fractional Programming (MOFLFP) problem. Yano and Sakawa [46] presented an interactive fuzzy decision making technique using bisection method and Simplex method to deal with MOFLFP problem. Sakawa et al. [47] developed a Pareto optimality for MOFLFP problems. Payan and Noora [48] used $\alpha$-cuts to convert the fuzzy parameters into interval parameters and the MOFLFP problem was finally reduced to a linear programming problem. Fuzzy Linear Fractional Programming (FLFP) containing all the variables and parameters as triangular fuzzy number has been solved by Pop and Stancu Minasian[49]. Ganesan and Veeramani [50] developed an approach to solve FLFP problem involving symmetric trapezoidal fuzzy numbers. Safaei [51] employed the decomposition method to solve FLFP problems.

Reviewing the literature of existing methods, it is observed that Taylor series based fuzzy mathematical programming approach to solve MOFLFP problems with fuzzy parameters is yet to appear.

Objective of the present study:

(i) To develop a robust, efficient and computationally simple technique to solve MOFLFP problem with fuzzy parameters using fuzzy mathematical programming and Taylor series approximation.

In this article, the MOFLFP problem is converted into a MOLFP problem by using $\alpha$-cuts to convert the fuzzy numbers into crisp numbers. The membership function associated with each objective function is converted into a linear function using first order Taylor series. Fuzzy mathematical programming technique is then employed to convert the MOLFP problem into a linear programming problem. The proposed approach is explained through a numerical example

The remaining article is as follows. Section 2 describes the preliminaries of fuzzy sets and fuzzy numbers. Section 3 deals with the formulation of MOFLFP problem. Section 4 devotes to present the proposed approach to solve MOFLFP problem with fuzzy parameters. Section 5 illustrates a numerical example to clarify of the method. Finally the paper ends with the concluding remarks in Section 6.

\section{PRELIMINARIES}

This section presents a brief outline of the basic notions of fuzzy sets, fuzzy numbers and different operations related to them.

Definition 1: Let $\mathrm{T}$ represent a universal set. Then, a fuzzy set [52] $\tilde{P}$ in $\mathrm{T}$ is a set of ordered pairs

$$
\tilde{P}=\left\{\left(t, \mu_{\tilde{P}}(t)\right) \mid t \in T\right\}
$$

where $\mu_{\tilde{P}}: T \rightarrow[0,1]$ denotes the membership function and is a way of expressing the degree to which $t \in \tilde{P}$.

Definition 2: $\tilde{P}$ is said to be a normal fuzzy set [52] if the condition $\mu_{\tilde{P}}(t)=1$ holds for at least one $t \in T$.

Definition 3: Support of a fuzzy set [52] $\tilde{P}$ is defined to be $\left\{t \in T \mid \mu_{\tilde{p}}(t)>0\right\}$.

Definition 4: The $\alpha$-cut or $\alpha$-level set of fuzzy set [52] $\tilde{P}$, denoted by $\tilde{P}_{\alpha}$ is a crisp set defined as $\left\{t: \mu_{\tilde{P}}(t) \geq \alpha\right\}$.

Definition 5: $\tilde{P}$ is referred to be a convex fuzzy set [52] if and only if the condition $\mu_{\tilde{P}}\left(\lambda t_{1}+(1-\lambda) t_{2}\right) \geq \min \left(\mu_{\tilde{P}}\left(t_{1}\right), \mu_{\tilde{P}}\left(t_{2}\right)\right)$ is satisfied by $\mu_{\tilde{P}}(t)$ for all $t_{1}, t_{2} \in T$ and for all $\lambda \in[0,1]$ where min stands for the minimum operator.

Definition 6: If R denotes the real line and $\tilde{P}$ is fuzzy subset of R, then $\tilde{P}$ is called a fuzzy number [52] if

$$
\begin{array}{ll}
\text { (i) } & \tilde{P} \text { is convex and normal. } \\
\text { (ii) } & \tilde{P} \text { possesses bounded support. }
\end{array}
$$

Definition 7: $\tilde{P}$ denotes a triangular fuzzy number [52] if its representation is in the form

$\tilde{P}=\left(p_{1}, p_{2}, p_{3}\right)$ with $p_{1} \leq p_{2} \leq p_{3}$ and the membership function of $\tilde{P}$ is constructed as follows:

$$
\mu_{\tilde{P}}(t)=\left\{\begin{array}{cc}
\frac{t-p_{1}}{p_{2}-p_{1}}, & p_{1} \leq t \leq p_{2} \\
\frac{p_{3}-t}{p_{3}-p_{2}}, & p_{2} \leq t \leq p_{3} \\
0, & \text { otherwise }
\end{array}\right.
$$

The $\alpha$-cut for a triangular fuzzy number $\tilde{P}=\left(p_{1}, p_{2}, p_{3}\right)$, is represented as

$\tilde{P}_{\alpha}=\left[P_{\alpha}^{L}, P_{\alpha}^{U}\right]=\left[p_{1}+\left(p_{2}-p_{1}\right) \alpha, p_{3}-\left(p_{3}-p_{2}\right) \alpha\right] \forall \alpha \in[0,1]$ and $P_{\alpha}^{L} \leq P_{\alpha}^{U}$.

A unique and complete representation of a fuzzy number can be made by its $\alpha$-cuts [53]. Hence $\alpha$-cuts have a great significance in the study of fuzzy numbers. $\alpha$ - cuts being closed intervals of real numbers, it enables us to define arithmetic operations on them and hence on the corresponding fuzzy number. Let $\tilde{P}=\left[P_{\alpha}^{L}, P_{\alpha}^{U}\right]$ and $\tilde{R}=\left[R_{\alpha}^{L}, R_{\alpha}^{U}\right]$ are considered as two arbitrary fuzzy numbers, then we can define 
the arithmetic operations [54] on $\tilde{P}$ and $\tilde{R}$ as follows:

(i) Addition: $(\tilde{P}+\tilde{R})_{\alpha}=\left[P_{\alpha}^{L}+R_{\alpha}^{L}, P_{\alpha}^{U}+R_{\alpha}^{U}\right]$

(ii) Scalar Multiplication: $(k \tilde{P})_{\alpha}=\left[k P_{\alpha}^{L}, k P_{\alpha}^{U}\right]$ if $k>0$, and $(k \tilde{P})_{\alpha}=\left[k P_{\alpha}^{U}, k P_{\alpha}^{L}\right]$, if $k<0$.

(iii) Multiplication:

$(\tilde{P} . \tilde{R})_{\alpha}=\left[\min \left(P_{\alpha}^{L} R_{\alpha}^{L}, P_{\alpha}^{L} R_{\alpha}^{U}, P_{\alpha}^{U} R_{\alpha}^{L}, P_{\alpha}^{U} R_{\alpha}^{U}\right)\right.$, $\left.\max \left(P_{\alpha}^{L} R_{\alpha}^{L}, P_{\alpha}^{L} R_{\alpha}^{U}, P_{\alpha}^{U} R_{\alpha}^{L}, P_{\alpha}^{U} R_{\alpha}^{U}\right)\right]$

(iv) Division:

$\left(\frac{\tilde{P}}{\tilde{R}}\right)_{\alpha}=\left[\begin{array}{c}\min \left(\frac{P_{\alpha}^{L}}{R_{\alpha}^{L}}, \frac{P_{\alpha}^{L}}{R_{\alpha}^{U}}, \frac{P_{\alpha}^{U}}{R_{\alpha}^{L}}, \frac{P_{\alpha}^{U}}{R_{\alpha}^{U}}\right) \\ \max \left(\frac{P_{\alpha}^{L}}{R_{\alpha}^{L}}, \frac{P_{\alpha}^{L}}{R_{\alpha}^{U}}, \frac{P_{\alpha}^{U}}{R_{\alpha}^{L}}, \frac{P_{\alpha}^{U}}{R_{\alpha}^{U}}\right)\end{array}\right]$

Definition 8: Let $\tilde{P}=\left(p_{1}, p_{2}, p_{3}\right)$ be a triangular fuzzy number. $\tilde{P}$ is termed non-negative if $p_{1} \geq 0$ and let $F\left(R^{+}\right)$ be defined as the collection of all such non-negative fuzzy numbers. $\tilde{P}$ is termed as a non-zero positive if $p_{1}>0$.

Assume that $F\left(R^{++}\right)$be the collection of all such non-zero positive fuzzy numbers.

\section{MULTI OBJECTIVE FUZZY LINEAR FRACTIONAL PROGRAMMING \\ PROBLEM}

Multi-Objective Fuzzy Linear Fractional Programming (MOFLFP) problem can be formulated as:

$\operatorname{Max} \tilde{Z}_{k}(t)=\frac{\sum_{j=1}^{s} \tilde{\alpha}_{k j} t_{j}+\tilde{c}_{k}}{\sum_{j=1}^{s} \tilde{\beta}_{k j} t_{j}+\tilde{d}_{k}}=\frac{\tilde{N}_{k}(t)}{\tilde{D}_{k}(t)}, k=1,2, \ldots, l$

subject to

$\left\{t \in R^{s} \mid \sum_{j=1}^{s} \tilde{a}_{i j} t_{j}\left(\begin{array}{l}\leq \\ = \\ \geq\end{array}\right) \tilde{p}_{i}, t \geq 0\right\}$

Equation (2) can be explicitly expressed as

$\sum_{j=1}^{s} \tilde{a}_{i j} t_{j} \leq \tilde{p}_{i}, i=1,2, \ldots, r_{1}$,

$\sum_{j=1}^{s} \tilde{a}_{i j} t_{j}=\tilde{p}_{i}, i=r_{1}+1, r_{1}+2, \ldots, r_{2}$,

$\sum_{j=1}^{s} \tilde{a}_{i j} t_{j} \geq \tilde{p}_{i}, i=r_{2}+1, r_{2}+2, \ldots, r$

$t_{j} \geq 0, j=1,2, \ldots, \mathrm{s}$

and $\tilde{\alpha}_{k j}, \tilde{\beta}_{k j}, \tilde{c}_{k}, \tilde{d}_{k}, \tilde{a}_{i j}, \tilde{p}_{i}$ are fuzzy numbers and $D_{k}(t)>0$, for all $t$, and $k=1,2, \ldots, l ; i=1,2, \ldots, r, j=1,2, \ldots, s$.

\section{FUZZY GOAL APPROACH FOR MOFLFP PROBLEM}

The linear fractional programming problem (1) can be transformed into a crisp linear fractional programming problem by taking the corresponding $\alpha$-cut in place of each triangular fuzzy number, and then choosing a particular value of $\alpha$. The conditions $N(t) \in F\left(R^{+}\right)$and $D(t) \in F\left(R^{++}\right)$are assumed for convenience. So for maximization problem, the coefficients of the numerator is replaced by the upper bound of the corresponding $\alpha$-level set and the coefficients of the denominator is to be substituted by the lower bound of the corresponding $\alpha$-level set [55], as follows:

$$
\begin{aligned}
\left(\tilde{Z}_{k}(t)\right)_{\alpha}= & \frac{\left(\sum_{j=1}^{s} \tilde{\alpha}_{k j} t_{j}+\tilde{c}_{k}\right)_{\alpha}^{U}}{\left(\sum_{j=1}^{s} \tilde{\beta}_{k j} t_{j}+\tilde{d}_{k}\right)_{\alpha}^{L}}=\frac{\left(\tilde{N}_{k}(t)\right)_{\alpha}^{U}}{\left(\tilde{D}_{k}(t)\right)_{\alpha}^{L}}, k=1,2, \ldots, l \\
= & \frac{\left(\tilde{\alpha}_{k 1}\right)_{\alpha}^{U} t_{1}+\left(\tilde{\alpha}_{k 2}\right)_{\alpha}^{U} t_{2}+\ldots+\left(\tilde{\alpha}_{k s}\right)_{\alpha}^{U} t_{s}+\left(\tilde{c}_{k}\right)_{\alpha}^{U}}{\left(\tilde{\beta}_{k 1}\right)_{\alpha}^{L} t_{1}+\left(\tilde{\beta}_{k 2}\right)_{\alpha}^{L} t_{2}+\ldots+\left(\tilde{\beta}_{k s}\right)_{\alpha}^{L} t_{s}+\left(\tilde{d}_{k}\right)_{\alpha}^{L}}
\end{aligned}
$$

Similarly, for minimization problem, the coefficients of the numerator is replaced by the lower bound of the corresponding $\alpha$-level set and the coefficients of the denominator is to be substituted by the upper bound of the corresponding $\alpha$-level set [55] as follows:

$$
\begin{aligned}
& \left(\tilde{Z}_{k}(t)\right)_{\alpha}=\frac{\left(\sum_{j=1}^{s} \tilde{\alpha}_{k j} t_{j}+\tilde{c}_{k}\right)_{\alpha}^{L}}{\left(\sum_{j=1}^{s} \tilde{\beta}_{k j} t_{j}+\tilde{d}_{k}\right)_{\alpha}^{U}}=\frac{\left(\tilde{N}_{k}(t)\right)_{\alpha}^{L}}{\left(\tilde{D}_{k}(t)\right)_{\alpha}^{U}}, k=1,2, \ldots, l \\
& =\frac{\left(\tilde{\alpha}_{k 1}\right)_{\alpha}^{L} t_{1}+\left(\tilde{\alpha}_{k 2}\right)_{\alpha}^{L} t_{2}+\ldots+\left(\tilde{\alpha}_{k s}\right)_{\alpha}^{L} t_{s}+\left(\tilde{c}_{k}\right)_{\alpha}^{L}}{\left(\tilde{\beta}_{k 1}\right)_{\alpha}^{U} t_{1}+\left(\tilde{\beta}_{k 2}\right)_{\alpha}^{U} t_{2}+\ldots+\left(\tilde{\beta}_{k s}\right)_{\alpha}^{U} t_{s}+\left(\tilde{d}_{k}\right)_{\alpha}^{U}}
\end{aligned}
$$

The inequality constraints of equation (3)

$\sum_{j=1}^{s} \tilde{a}_{i j} t_{j} \leq \tilde{p}_{i}, i=1,2, \ldots, r_{1}$,

$\sum_{j=1}^{s} \tilde{a}_{i j} t_{j} \geq \tilde{p}_{i}, i=r_{2}+1, r_{2}+2, \ldots, r$

are replaced by the following inequalities

$\sum_{j=1}^{s}\left(\tilde{a}_{i j}\right)_{\alpha}^{L} t_{j} \leq\left(\tilde{p}_{i}\right)_{\alpha}^{U}, i=1,2, \ldots, r_{1}$,

$\sum_{j=1}^{s}\left(\tilde{a}_{i j}\right)_{\alpha}^{U} t_{j} \geq\left(\tilde{p}_{i}\right)_{\alpha}^{L}, i=r_{2}+1, r_{2}+2, \ldots, r$

The equality constraint of equation (3)

$\sum_{j=1}^{s} \tilde{a}_{i j} t_{j}=\tilde{p}_{i}, i=r_{1}+1, r_{1}+2, \ldots, r_{2}$

is replaced by the following two inequalities [56]:

$$
\begin{aligned}
& \sum_{j=1}^{s}\left(\tilde{a}_{i j}\right)_{\alpha}^{L} t_{j} \leq\left(\tilde{p}_{i}\right)_{\alpha}^{U}, i=r_{1}+1, r_{1}+2, \ldots, r_{2}, \\
& \sum_{j=1}^{s}\left(\tilde{a}_{i j}\right)_{\alpha}^{U} t_{j} \geq\left(\tilde{p}_{i}\right)_{\alpha}^{L}, i=r_{1}+1, r_{1}+2, \ldots, r_{2} .
\end{aligned}
$$

So equation (3) can be substituted by a set of reduced constraints [56] as follows: 
$\left.\begin{array}{l}\sum_{j=1}^{s}\left(\tilde{a}_{i j}\right)_{\alpha}^{L} t_{j} \leq\left(\tilde{p}_{i}\right)_{\alpha}^{U}, i=1,2, \ldots, r_{1}, \\ \sum_{j=1}^{s}\left(\tilde{a}_{i j}\right)_{\alpha}^{L} t_{j} \leq\left(\tilde{p}_{i}\right)_{\alpha}^{U}, i=r_{1}+1, r_{1}+2, \ldots, r_{2}, \\ \sum_{j=1}^{s}\left(\tilde{a}_{i j}\right)_{\alpha}^{U} t_{j} \geq\left(\tilde{p}_{i}\right)_{\alpha}^{L}, i=r_{1}+1, r_{1}+2, \ldots, r_{2}, \\ \sum_{j=1}^{s}\left(\tilde{a}_{i j}\right)_{\alpha}^{U} t_{j} \geq\left(\tilde{p}_{i}\right)_{\alpha}^{L}, i=r_{2}+1, r_{2}+2, \ldots, r, \\ \mathrm{t} \geq 0 .\end{array}\right\}$

where the lower bounds $\left(p_{i}\right)_{\alpha}^{L},\left(a_{i j}\right)_{\alpha}^{L}$ and the upper bounds $\left(p_{i}\right)_{\alpha}^{U},\left(a_{i j}\right)_{\alpha}^{U}$ of the coefficients are set to have the largest feasible region so that we can most certainly find the compromise solutions of objective functions.

We denote the system constraints (6) as $\mathrm{S}^{\prime}$.

\subsection{Construction of Membership function for solving MOFLFP Problem}

We consider the maximum and minimum value of each objective function as the acceptable upper and lower tolerance limit respectively to construct the membership function of the objective function.

Let $Z_{k}^{B}=\max _{t \in S^{\prime}} \tilde{Z}_{k}(t)$ and $Z_{k}^{W}=\min _{t \in S^{\prime}} \tilde{Z}_{k}(t) \quad k=1,2, \ldots, l$ denotes the best value and the worst value of $\mathrm{k}$-th objective function respectively where the conditions of non-negativity and the constraint equations are satisfied by $t$.

The membership function of $\mathrm{k}$-th objective function is constructed as:

$$
\mu_{k}(t)= \begin{cases}1, & \text { if } \tilde{Z}_{k}(t) \geq Z_{k}^{B} \\ \frac{\tilde{Z}_{k}(t)-Z_{k}^{W}}{Z_{k}^{B}-Z_{k}^{W}}, & \text { if } Z_{k}^{W} \leq \tilde{Z}_{k}(t) \leq Z_{k}^{B} \\ 0, & \text { if } \tilde{Z}_{k}(t) \leq Z_{k}^{W}\end{cases}
$$

We assume that each objective function $\tilde{Z}_{k}(t)$ possesses continuous partial derivatives of order $(s+1)$ or less than that on the feasible region $\mathrm{S}^{\prime}$, where $\mathrm{S}^{\prime}$ denotes the region represented by the equation (6).

So the membership function $\mu_{k}(t)$ corresponding to the objective function $\tilde{Z}_{k}(t)$ bears similar properties in the feasible region $S^{\prime}$

So the problem (1) gets reduced to the following problem:

$\max \mu_{k}(t), k=1,2, \ldots, l$

subject to

$t \in S^{\prime}$

\subsection{Linearization of Membership Functions with the Help of First Order Taylor Series Approximation}

Assume that the individual best solution for problem (8) exists at $t_{k}^{*}=\left(t_{k 1}^{*}, t_{k 2}^{*}, \ldots . ., t_{k s}^{*}\right)$ subject to the system constraints $\mathrm{S}^{\prime}$.

We apply first order Taylor series to convert the membership function $\mu_{k}(t)$ into an equivalent linear form. The reduced membership function can be written as:

$$
\left.\begin{array}{rl}
\mu_{k}(t) & \cong \mu_{k}\left(t_{k}^{\bullet}\right)+\left(t_{1}-t_{k 1}^{\bullet}\right)\left(\frac{\partial}{\partial t_{1}} \mu_{k}(t)\right)_{a t t=t_{k}^{\bullet}} \\
& +\left(t_{2}-t_{k 2}^{\bullet}\right)\left(\frac{\partial}{\partial t_{2}} \mu_{k}(t)\right)_{a t ~}=t_{k}^{\cdot}+\ldots \\
& +\left(t_{s}-t_{k s}^{\bullet}\right)\left(\frac{\partial}{\partial t_{s}} \mu_{k}(t)\right)_{a t ~}=t_{k 1}^{\cdot}
\end{array}\right\}
$$

\subsection{Fuzzy Mathematical Programming Formulation of MOFLFP problem}

Using (9), the problem (8) can be converted to an equivalent linear programming problem as follows:

$\operatorname{Max} \theta$

subject to

$t \in S^{\prime}$

$\hat{\mu}_{k}(t) \geq \theta \quad k=1,2, \ldots ., l$

$0 \leq \theta \leq 1$.

Model (10) can be expressed in an explicit form as:

$\operatorname{Max} \theta$

subject to

$t \in S^{\prime}$

$\mu_{k}\left(t^{*}\right)+\left(t_{1}-t_{k 1}^{*}\right)\left(\frac{\partial}{\partial t_{1}} \mu_{k}(t)\right)_{a t t=t_{k}^{*}}$

$+\left(t_{2}-t_{k 2}^{*}\right)\left(\frac{\partial}{\partial t_{2}} \mu_{k}(t)\right)_{a t ~}=t_{k}^{*}+\ldots$

$+\left(t_{S}-t_{k S}^{*}\right)\left(\frac{\partial}{\partial t_{S}} \mu_{k}(t)\right)_{a t t=t_{k}^{*}} \geq \theta, k=1,2, \ldots ., l ;$

$0 \leq \theta \leq 1$.

\subsection{Algorithm for solving MOFLFP problem}

The above process is summed up in the following algorithm.

Step 1: Replace the fuzzy parameters with triangular fuzzy numbers

Step 2: Replace the fuzzy numbers with $\alpha$-cuts according to equation 4- 6.

Step 3: Choose a value of $\alpha$ and construct the problem as a crisp problem.

Step 4: Determine the individual maximum and minimum value of each objective function.

Step 5: Determine the membership functions according to equation 7 .

Step 6: Linearize each membership function using first order Taylor series about the point at which individual best solution is obtained.

Step 7: Formulation of fuzzy programming model of MOFLFP using linearized membership function.

Step 8: Obtain the solution of the problem in step 7 by solving the fuzzy mathematical programming model. 


\section{NUMERICAL EXAMPLE}

We consider the following problem studied by Payan and Noora [48].

$\max Z_{1}=\frac{\tilde{2} x_{1}+4 x_{2}+\tilde{5}}{\tilde{2} x_{1}+6}$

$\max Z_{2}=\frac{x_{1}+\tilde{6} x_{2}+50}{x_{1}+\tilde{1} x_{2}+\tilde{8}}$

subject to

$$
\left.\begin{array}{l}
\tilde{2} x_{1}+2 x_{2} \leq 1 \tilde{40} 0 \\
x_{2} \geq \tilde{8} \\
x_{1} \geq \tilde{16} \\
x_{1}, x_{2} \geq 0
\end{array}\right\}
$$

where the fuzzy parameters assume the triangular fuzzy numbers as the following form:

$\tilde{5}=(3,5,7), \tilde{1}=(0,1,2), \tilde{2}=(1,2,3), 140=(100,140,180)$,

$\tilde{8}=(6,8,10), \tilde{16}=(6,16,20), \tilde{6}=(4,6,8)$

Using equation (4) and equation (6), problem (12) can be written as:

$\max Z_{1}=\frac{(3-\alpha) x_{1}+4 x_{2}+(7-2 \alpha)}{(1+\alpha) x_{1}+6}$

$\max Z_{2}=\frac{x_{1}+(8-2 \alpha) x_{2}+50}{x_{1}+\alpha x_{2}+(6+2 \alpha)}$

subject to

$(1+\alpha) x_{1}+2 x_{2} \leq(180-40 \alpha)$

$x_{2} \geq(6+2 \alpha)$

$x_{1} \geq(6+10 \alpha)$

$x_{1}, x_{2} \geq 0$

Considering $\alpha=0.5$ the problem (13) is transformed into a crisp linear fractional programming problem as follows:

$\max Z_{1}=\frac{2.5 x_{1}+4 x_{2}+6}{1.5 x_{1}+6}$
$\max Z_{2}=\frac{x_{1}+7 x_{2}+50}{x_{1}+0.5 x_{2}+7}$

subject to

$1.5 x_{1}+2 x_{2} \leq 160$

$x_{2} \geq 7$

$x_{1} \geq 11$

$x_{1}, x_{2} \geq 0$

Solving problem (14) we find the individual best solutions as $Z_{1}^{B}=14.24$ at $x_{1}=11.00, x_{2}=71.75$ and $Z_{2}^{B}=10.455$ at $x_{1}=10.99, x_{2}=71.75$. The individual worst solutions are found as $Z_{1}^{W}=1.825$ at $x_{1}=97.32, x_{2}=6.99$ and $Z_{2}^{W}=1.821$ at $x_{1}=97.334, x_{2}=7.00$.

So the membership functions are constructed as:

$$
\begin{aligned}
& \mu_{1}(x)=\frac{Z_{1}(x)-1.825}{14.24-1.825}=\frac{\frac{2.5 x_{1}+4 x_{2}+6}{1.5 x_{1}+6}-1.825}{14.24-1.825} \\
& \mu_{2}(x)=\frac{Z_{2}(x)-1.821}{10.455-1.821}=\frac{\frac{x_{1}+7 x_{2}+50}{x_{1}+0.5 x_{2}+7}-1.821}{10.455-1.821}
\end{aligned}
$$

Figures 1 and 2 depict the graph of the membership functions of the objective functions $\mathrm{Z}_{1}$ and $\mathrm{Z}_{2}$.

The membership functions $\mu_{1}(x)$ and $\mu_{2}(x)$ are converted into linear functions by employing the first order Taylor series about the points at which the best solutions are obtained, obtained, that is, $(11.00,71.75)$ and $(10.99,71.75)$ respectively.

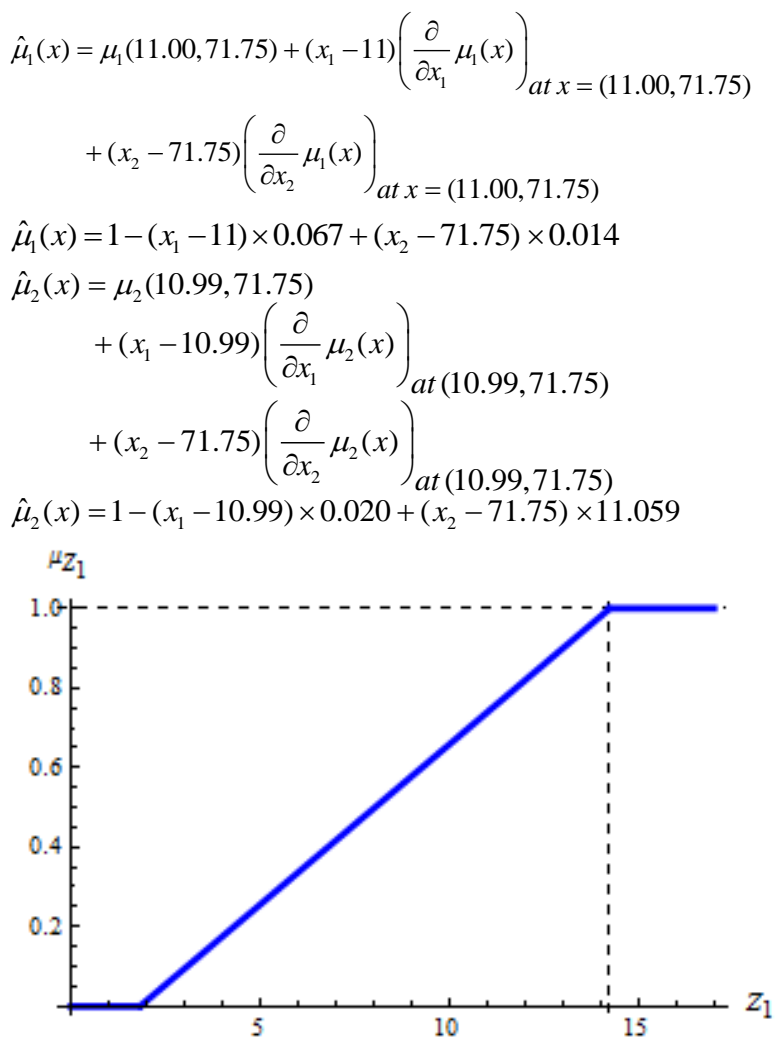

Figure 1: Graphical representation of membership function of $\mathrm{Z}_{1}$

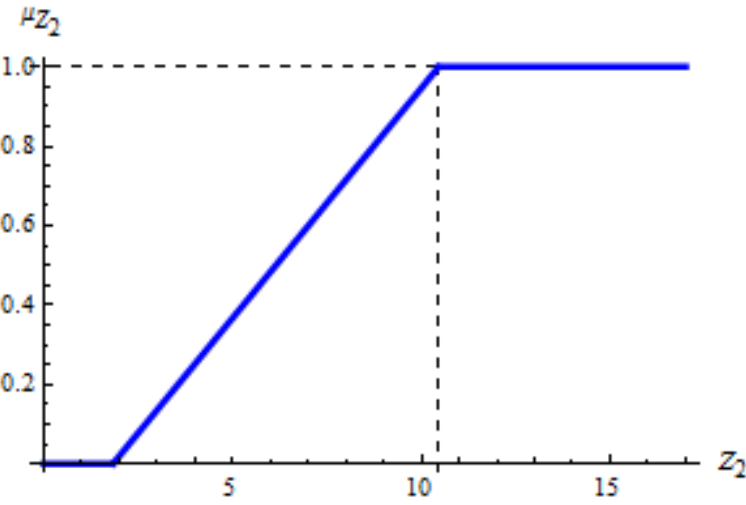

Figure 2: Graphical representation of membership function of $\mathbf{Z}_{2}$ 
The fuzzy mathematical programming model can be presented as:

$\operatorname{Max} \theta$

subject to

$1-\left(x_{1}-11\right) \times 0.067+\left(x_{2}-71.75\right) \times 0.014 \geq \theta$

$1-\left(x_{1}-10.99\right) \times 0.020+\left(x_{2}-71.75\right) \times 11.059 \geq \theta$

$1.5 x_{1}+2 x_{2} \leq 160$

$x_{2} \geq 7$

$x_{1} \geq 11$

$0 \leq \theta \leq 1$

$x_{1}, x_{2} \geq 0$

The problem (19) provides the solution $Z_{1}=14.24, Z_{2}=10.455$ at $x_{1}=11.00, x_{2}=71.75$. The resulting membership values are $\mu_{1}=1, \mu_{2}=1$.

Table 1. Optimal solutions of the numerical example 1 for different values of $\alpha$

\begin{tabular}{|c|c|c|c|c|}
\hline$\alpha$ & $x_{1}$ & $x_{2}$ & $Z_{1}$ & $Z_{2}$ \\
\hline 1.00 & 16 & 54 & 6.658 & 5 \\
\hline 0.50 & 11 & 71.75 & 14.24 & 10.455 \\
\hline 0.25 & 8.5 & 79.68 & 21 & 18.79 \\
\hline 0.00 & 6 & 87 & 31 & 62.67 \\
\hline
\end{tabular}

It is observed that the solutions obtained by Yano and Sakawa[46] and Payan and Noora [48] are the same as obtained from the proposed method.

It is to be noted that the methodology proposed in the study is computationally much simpler and easy to implement than the methods proposed in [46, 48]. In [48], the MOFLFP problem was converted into MOLFP with interval parameters and then into MOLFP. This MOLFP was finally transformed into linear programming (LP) problem with interval parameter. This was divided into two LPs and the optimal solution was obtained based on their solutions. Yano and Sakawa [46] solved this problem by non-linear programming technique which used bisection method and simplex method. The problem was solved through iterations. Our method uses upper bound of $\alpha$-cut value for the numerator and lower bound of the $\alpha$ - cut value for the denominator in a fuzzy fractional linear programming problem of maximization type . Thus the problem is converted into a crisp linear-fractional problem very easily which is solved by using goal programming. All the problems have been solved by LINGO version 17

\section{CONCLUSION}

In this study, we present an alternative fuzzy programming model to solve MOFLFP problems. In the proposed approach, the membership functions are converted into linear form using first order Taylor polynomial series approximation. Each objective function with fuzzy parameters is transformed into crisp objective functions using $\alpha$-cut of the fuzzy numbers. Then fuzzy programming method has been used to solve the MOFLFP problem using only the negative deviational variables. So it can be concluded that the method developed in this study is robust, computationally simpler and easy to implement than the existing methods [46, 48]. A numerical illustrative example has been solved so demonstrate the proposed approach. The proposed method can be extended to solve bi-level multi objective and multi-level multi-objective linear fractional programming problems with fuzzy parameters. The proposed concept is expected to be helpful in optimizing linear fractional problems with fuzzy parameters in real life problems such as in agriculture, inventory problems, production planning, transportation problem etc.

\section{ACKNOWLEDGEMENTS}

The second author greatly acknowledges the financial support provided by Department of Science and Technology, INSPIRE fellowship (Govt. of India) to conduct the study.

\section{REFERENCES}

[1] Isbell, J. R., and Marlow, W. H. 1956. Attrition games. Naval Research Logistics Quarterly 3 (1-2), 71-94.

[2] Charnes, A., and Cooper, W. W. 1962. Programming with linear fractional functionals. Naval Research Logistics Quarterly 9 (3-4), 181-186.

[3] Dinklebach, W. 1967. On nonlinear fractional programming. Management Science 13 (7), 492-498.

[4] Schaible, S. 1976. Fractional programming. I, duality. Management Science 22 (8), 858-867.

[5] Gilmore, P. C., and Gomory, R. E. 1963. A linear programming approach to the cutting stock problem-Part II. Operations Research 11 (6), 863-888.

[6] Swarup, K. 1965. Linear fractional functional programming. Operations Research 13 (6), 1029-1036.

[7] Kornbluth, J. S. H., and Steur, R. E. 1981. Multiple objective linear fractional programming. Management Science 27 (9), 1024-1039.

[8] Nykowski, I., and Zolkiewski, Z. 1985. A compromise procedure for the multiple objective linear fractional programming problem. European Journal of Operational Research 19 (1), 91-97.

[9] Dey, P. P., and Pramanik, S. 2011. Goal programming approach to linear fractional bilevel programming problem based on Taylor series approximation. International Journal of Pure and Applied Sciences and Technology 6(2), 115-123.

[10] Zadeh, L. A. 1965. Fuzzy sets. Information and Control 8(3), 338-353.

[11] Luhandjula, M. K. 1984. Fuzzy approaches for multiple objective linear fractional optimization. Fuzzy Sets and Systems 13 (1), 11-23.

[12] Zadeh, L. A. 1975a. The concept of a linguistic variable and its application to approximate reasoning, Part III. Information Sciences 9 (1), 43-80.

[13] Zadeh, L. A. 1975b. The concept of a linguistic variable and its application to approximate reasoning, Part II. Information Sciences 8 (4), 301-352.

[14] Zadeh, L. A. 1975c. The concept of a linguistic variable and its application to approximate reasoning, Part I. Information Sciences 8 (3), 199-244.

[15] Pal, B. B., Moitra, B. N., and Maulik, U. 2003. A goal programming procedure for fuzzy multiobjective linear fractional programming problem. Fuzzy Sets and Systems 139 (2), 395-405. 
[16] Sakawa, M., and Yano, H. 1988. An interactive fuzzy satisficing method for multiobjective linear fractional programming problems. Fuzzy Sets and Systems 28 (2), 129-144.

[17] Dutta, D., Tiwari, R. N., and Rao, J. R. 1992. Multiple objective linear fractional programming - a fuzzy set theoretic approach. Fuzzy Sets and Systems 52 (1), 3945 .

[18] Chakraborty, M., and Gupta, S. 2002. Fuzzy mathematical programming for multi objective linear fractional programming problem. Fuzzy Sets and Systems 125 (3), 335-342.

[19] Guzel, N., and Sivri, M. 2005. Taylor series solution to multi-objective linear fractional programming problem. Trakya University Journal Sciences 6 (2), 80-87.

[20] Toksari, D. M. 2008. Taylor series approach to fuzzy multiobjective linear fractional programming. Information Sciences 178 (4), 1189-1204.

[21] Pramanik, S., and Roy, T. K. 2005. A fuzzy goal programming approach for multi-objective capacitated transportation problem. Tamsui Oxford Journal of Management Sciences 21(1), 75-88.

[22] Pramanik, S., and Roy, T. K. 2007. Fuzzy goal programming approach to multilevel programming problems. European Journal of Operational Research 176 (2) $1151-1166$

[23] Pramanik, S., \& Roy, T. K. 2006. A fuzzy goal programming technique for solving multi-objective transportation problem. Tamsui Oxford Journal of Management Sciences 22 (1), 67-89.

[24] Pramanik, S., \& Roy, T. K. 2008. Multiobjective transportation model with fuzzy parameters: a priority based fuzzy goal programming. Journal of Transportation Systems Engineering and Information Technology 8 (3), 40-48.

[25] Pramanik, S. 2012. Bilevel programming problem with fuzzy parameter: a fuzzy goal programming approach. Journal of Applied Quantitative Methods 7(1), 09-24.

[26] Pramanik, S. 2015. Multilevel programming problems with fuzzy parameters: a fuzzy goal programming approach. International Journal of Computer Applications 122 (21), 34-41.

[27] Pramanik, S., and Dey, P.P. 2011. Bi-level multiobjective programming problem with fuzzy parameters. International Journal of Computer Applications 30 (10) 13-20.

[28] Pramanik, S., Dey, P. P., and Giri. B. C. 2011. Decentralized bilevel multiobjective programming problem with fuzzy parameters based on fuzzy goal programming. Bulletin of Calcutta Mathematical Society 103 (5), 381-390.

[29] Pramanik, S., and Dey, P. P. 2011. Multi-objective linear fractional programming problem based on fuzzy goal programming. International Journal of Mathematical Archive 2 (10), 1875-1881

[30] Pramanik, S., and Dey, P.P. 2011. A priority based fuzzy goal programming to multi-objective linear fractional programming problem. International Journal of Computer Applications 30 (10), 1-6.
[31] Pramanik, S., and Dey, P.P. 2011. Bi-level linear fractional programming problem based on fuzzy goal programming approach. International Journal of Computer Applications 25 (11), 34-40.

[32] Dey, P. P., Pramanik, S., and Giri, B.C.2013. Fuzzy goal programming algorithm for solving bi-level multiobjective linear fractional programming problems, International Journal of Mathematical Archive 4(8), 154 161.

[33] Pramanik, S., Dey, P. P., and Roy, T. K. 2012. Fuzzy goal programming approach to linear fractional bilevel decentralized programming problem based on Taylor series approximation. The Journal of Fuzzy Mathematics 20 (1), 231- 238.

[34] Dey, P.P., Pramanik, S., and Giri, B. C. 2014. Multilevel fractional programming problem based on fuzzy goal programming. International Journal of Innovative research in Technology \& Science 2(4), 17-26.

[35] Dey, P. P., Pramanik, S., \&Giri, B. C. 2014. TOPSIS approach to linear fractional bi-level MODM problem based on fuzzy goal programming, Journal of Industrial and Engineering International 10(4), 173-184.

[36] Banerjee, D., \& Pramanik, S. 2012. Goal programming approach to chance constrained multi-objective linear fractional programming problem based on Taylor's series approximation. International Journal of Computers \& Technology 2(2), 77-80.

[37] Pramanik, S., and Dey, P.P. 2011. Multi-objective linear plus linear fractional programming problem based on Taylor series approximation. International Journal of Computer Applications 32 (8), 61-68.

[38] Pramanik, S., Banerjee, D., and Giri, B.C. 2015. Multilevel multi-objective linear plus linear fractional programming problem based on FGP approach. International Journal of Innovative Science Engineering and Technology 2 (6), 153-160.

[39] Banerjee, D., \& Pramanik, S. 2012. Chance constrained multi-objective linear plus linear fractional programming problem based on Taylor's series approximation. International Journal of Engineering Research and Development 1(3) 55-62.

[40] Pramanik, S., Banerjee, D., \& Giri, B.C. 2012. Chance constrained linear plus linear fractional bi-level programming problem. International Journal of Computer Applications 56(16), 34-39.

[41] Pramanik, S., and Dey, P.P. 2011. Multi-objective quadratic programming problem based on fuzzy goal programming. International Journal of Pure and Applied Sciences and Technology 6(1), 45-53.

[42] Pramanik, S., and Dey, P.P. 2011. Multi-objective quadratic programming problem: a priority based fuzzy goal programming. International Journal of Computer Applications 26 (10), 30-35.

[43] Pramanik, S., and Dey, P.P. 2011. Quadratic bi-level programming problem based on fuzzy goal programming approach. International Journal of Software Engineering \& Application 2(4), 41-59.

[44] Pramanik, S., Dey, P. P., and Giri, B.C. 2011. Fuzzy goal programming approach to quadratic bi-level multi- 
objective programming problem. International Journal of Computer Applications 29 (6), 09-14.

[45] Pramanik, S., \& Banerjee, D. 2012. Chance constrained quadratic bi-level programming problem. International Journal of Modern Engineering Research 2(4), 24172424.

[46] Yano, H., and Sakawa, M. 1989. Interactive fuzzy decision making for generalized multiobjective linear fractional programming problems with fuzzy parameters. Fuzzy Sets and Systems 32 (3), 245-261.

[47] Sakawa, M., Yano, H., and Takahashi, J. 1992. Pareto optimality for multionjective linear fractional programming problems with fuzzy parameters. Information Sciences 63 (1-2), 33-53.

[48] Payan, A., and Noora, A. A. 2014. A linear modeling to solve multi-objective linear fractional programming problem with fuzzy parameters. International Journal Mathematical Modelling and Numerical Optimisation 5 (3), 210-228.

[49] Pop, B., and Stancu-Minasian, I. M. 2008. A method of solving fully fuzzified linear fractional programming problems. Journal of Applied Mathematics and Computing 27 (1-2), 227-242.
[50] Ganesan, K., and Veeramani, P. 2006. Fuzzy linear programs with trapezoidal fuzzy numbers. Annals of Operations Research 143, 305-315.

[51] Safaei, N. 2014. A new method for solving fully fuzzy linear fractional programming with triangular fuzzy numbers. Applied Mathematics and Computational Intelligence 3 (1), 273-281.

[52] Zimmermann, H. J. 1991. Fuzzy sets theory and its applications. Kluwer Academic, Boston.

[53] Sakawa, M. 1993. Fuzzy sets and interactive multiobjective optimization. Plenum Press, New York.

[54] Kauffmann, A., and Gupta, M. M. 1991 Introduction to fuzzy Arithmetic: Theory and Applications. Van Nostrand Reinhold, New York.

[55] Mehra, A., Chandra, S., and Bector, C. R. 2007. Acceptable optimality in linear fractional programming with fuzzy coefficients. Fuzzy Optimization Decision Making 6 (1), 5-16.

[56] Lee, E. S., and Li, R. J. 1993. Fuzzy multiple objective programming with Pareto optimum. Fuzzy Sets and Systems 53 (3), 275-288. 